\title{
Intravesical atropine and suppression of detrusor hypercontractility in the neuropathic bladder. A preliminary study
}

\author{
S Glickman ${ }^{1}, \mathrm{~N}$ Tsokkos ${ }^{2}$ and PJR Shah ${ }^{3}$ \\ ${ }^{1}$ Formerly Senior Registrar in Rehabilitation Medicine, Royal National Orthopaedic Hospital, Stanmore. \\ Currently Consultant in Rehabilitation Medicine, Charing Cross Hospital; ${ }^{2}$ Formerly Senior Registrar in Urology, \\ St Peter's Hospital, London. Currently Consultant Gynaecologist, Perth, Australia; ${ }^{3}$ Consultant Urologist, Spinal \\ Injuries Unit, Royal National Orthopaedic Hospital, Stanmore and Senior Lecturer, Institute of Urology, London
}

\begin{abstract}
Twelve patients with detrusor hypercontractility secondary to spinal cord lesions were treated with intravesical atropine sulphate. Five patients expelled the solution almost immediately. In the seven patients who retained the solution the cystometric capacity increased by a mean of $301 \mathrm{ml}(P<0.01)$. The volume at which an unstable contraction occurred increased by a mean of $190 \mathrm{ml}(P<0.05)$ and the volume at which a urinary leak occurred increased by a mean of $297 \mathrm{ml}(P<0.05)$. The maximum hyperreflexic detrusor contraction pressure was reduced by a mean of $24 \mathrm{~cm}$ water $(P<0.05)$. No significant reduction in end fill pressure or compliance was observed though the increase in compliance approached significance. No side effects were observed and no differences were observed in pupillary reactions, blood pressure or pulse after the instillation of atropine.
\end{abstract}

Keywords: intravesical; atropine; detrusor; neuropathic bladder; anticholinergic drugs

\section{Introduction}

Detrusor hypercontractility is frequently associated with the neuropathic bladder of patients with neurological disorders, and in particular spinal cord lesions above the sacral reflex arc. Because of repeated and sustained high pressure detrusor contractions, urinary incontinence, ureteric reflux and obstruction, pyelonephritis and long term renal damage become significant problems for these patients.

Anticholinergic drugs may be used to suppress detrusor contractions but are associated with a substantial morbidity from the side effects of thirst, dry mouth, blurred vision, glaucoma, constipation, dry skin, arrhythmias and confusion. The anticholinergic oxybutynin chloride used intravesically has been reported as being effective in animal experiments ${ }^{1-3}$ and in humans ${ }^{4-8}$ in suppressing detrusor contraction with a very low incidence of side effects. However oxybutynin chloride is expensive to produce and is currently only available for restricted use in its intravesical form on a named-patient basis. Clinical trials of intravesical oxybutynin are currently underway.

Atropine sulphate is a widely used anticholinergic agent which is available in liquid form. However when given intravenously, atropine only partly inhibits detrusor contractions at doses which produce significant side effects; ${ }^{9}$ though a dose of $0.5 \mathrm{mg}$ intravenously may produce side effects, doses up to $200 \mathrm{mg}$ have been

Correspondence: Mr PJR Shah, Institute of Urology and Nephrology, 48 Riding House Street, London W1P 7PN safely used therapeutically. ${ }^{9}$ Atropine is largely excreted in urine $(56-58 \%)$ but there is little information about possible reabsorption from the bladder. ${ }^{10}$ It is possible to assume that atropine is safe to instil intravesically but the degree of systemic uptake is unknown. It has been used in the experimental animal with benefit. ${ }^{1-3}$

The aim of this preliminary study was to investigate the efficacy and the side effects of the instillation of atropine sulphate into the neuropathic bladder and to consider its effects. This could possibly indicate a clinical use for atropine in the treatment of detrusor hyperreflexia.

\section{Patients and methods}

Patients with a neuropathic type of bladder secondary to spinal cord lesions who had not received an anticholinergic agent for more than 2 weeks were recruited for the study. Informed consent was obtained. Atropine sulphate was diluted with normal saline to a volume of $20 \mathrm{ml}$ and instilled intravesically. The instillation was given at ambient room temperature. Initially patients were given incremental doses of atropine sulphate ranging from $0.6 \mathrm{mg}$ to $10.2 \mathrm{mg}$ and assessed for side effects. Subsequently patients received a dose of $6 \mathrm{mg}$ atropine sulphate intravesically and were then further assessed. All of these patients had a baseline cystometrogram performed within 4 months of the study or had a cystometrogram performed on the day of the atropine study prior to the instillation of the atropine. A cystometrogram was 
performed between 2 and $4 \mathrm{~h}$ after the instillation of the atropine. All cystometrograms were performed by slow filling of the bladder $\left(10 \mathrm{ml} \mathrm{s}^{-1}\right)$ using saline at room temperature with the patient in the supine position. The bladder was drained of residual urine at the commencement of the cystometrogram in order to standardise the method. Subtraction cystometrograms were performed using a Disa System 2100 (no longer manufactured) and fluid filled catheters connected to Dantec 22K12 pressure transducers (Dantec Electronics Ltd, Bristol, UK). Methods, definitions and units conform to the standards recommended by the International Continence Society except where specifically noted. ${ }^{11}$ Patients were monitored for side effects by measuring pulse, blood pressure, pupillary reflexes and questioning about the presence of dry mouth and blurred vision. Outcome was assessed by measuring maximum cystometric capacity, volume at which the first detrusor contraction occurred, volume at which leakage first occurred, end fill pressure and the pressure of the maximum detrusor contraction. Subsequently compliance was calculated using the formula volume change $(\Delta \mathrm{V})$ divided by the change in detrusor pressure $(\Delta \mathrm{Pdet})$.

\section{Results}

No side effects were observed in the first four patients given the incremental doses of atropine sulphate intravesically. Twelve patients received atropine instillation and were further assessed with pre- and postatropine cystometrograms. There were 10 males and two females. Ten patients had spinal lesions secondary to trauma. One patient had transverse myelitis and one patient had a spinal haemangioma. All lesions were above L1. Seven patients were being managed with intermittent self catheterisation, three with suprapubic catheterisation, and one with an indwelling urethral catheter. All but two patients were experiencing episodes of incontinence due to hyperreflexic detrusor contractions. Of the patients who were not experiencing incontinence one had a permanent indwelling urethral catheter and the other had a suprapubic catheter. The mean age was 27.5 (range 18-40; SE 1.9). Eight patients had developed the spinal cord lesion within 4 months of the test, a further three within 24 months, and one was 96 months since initial presentation.

Five patients experienced detrusor contractions within minutes of the instillation resulting in expulsion of the solution. This is most likely due to the instillation of the solution at room temperature. Four of these went on to have urodynamics but showed no significant differences between the pre-and post-atropine urodynamics. The other patient refused a second cystometrogram.

The seven patients who retained the atropine showed several statistically and clinically significant differences between the pre- and post-atropine urodynamics (Table 1). The volume at which a detrusor contraction occurred increased by a mean of $190 \mathrm{ml}(P<0.05)$ and the volume at which a urinary leak occurred increased by a mean of $297 \mathrm{ml}(P<0.05)$. The cystometriccapacity increased by a mean of $301 \mathrm{ml}(P<0.01)$ (Figure 1). The maximum detrusor contraction pressure was reduced by a mean of $24 \mathrm{~cm}$ of water $(P<0.05)$ (Figure 2). A non-significant reduction in detrusor end fill pressure and compliance were observed although the increase in compliance approached significance.

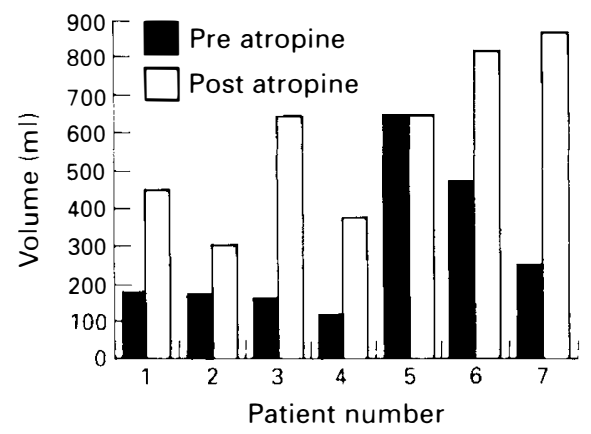

Figure 1 Cystometric capacity $(P<0.01)$.

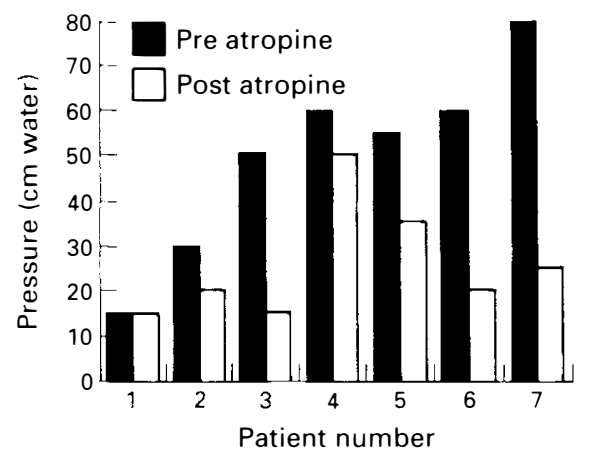

Figure 2 Maximum detrusor capacity $(P<0.05)$

Table 1 The differences between the pre- and the post-atropine urodynamics

\begin{tabular}{lcccc}
\hline & $\begin{array}{c}\text { Pre atropine } \\
\text { Mean } \pm \text { SE }\end{array}$ & $\begin{array}{c}\text { Post atropine } \\
\text { Mean } \pm \text { SE }\end{array}$ & $\begin{array}{c}\text { Mean difference } \\
\pm S E\end{array}$ \\
\hline Volume of first unstable contraction (ml) & $199 \pm 43$ & $389 \pm 68$ & $190 \pm 71.7$ & $<0.05$ \\
Volume of first leakage (ml) & $249 \pm 59$ & $546 \pm 104$ & $297 \pm 83$ & $<0.05$ \\
Cystometric capacity (ml) & $256 \pm 74$ & $587 \pm 82$ & $301 \pm 79$ & $<0.001$ \\
Maximum unstable contraction (cm water) & $50 \pm 8$ & $26 \pm 4$ & $24 \pm 7$ & $<0.05$ \\
Endfill pressure (cm water) & $18 \pm 6$ & $13 \pm 3$ & $6 \pm 6$ & $>0.05$ \\
Compliance (ml)/cm water & $36 \pm 16$ & $78 \pm 28$ & $39 \pm 24$ & $>0.05$ \\
\hline
\end{tabular}


No side effects were observed and no differences were observed in pupillary reactions, blood pressure or pulse after the instillation of atropine.

\section{Discussion}

Detrusor activity is largely under the control of the parasympathetic nervous system. The principle peripheral neurotransmitter involved is acetylcholine which acts on nicotinic receptors at the post-ganglionic cell body and muscarinic receptors at the effector sites on the smooth muscle cells of the detrusor muscle. Stimulation of these effector sites by acetylcholine leads to detrusor contraction.

Atropine is one of the belladonna alkaloids which exhibits antimuscarinic effects by competitive antagonism to acetylcholine at the effector cell level. When administered orally or parenterally its clinical usefulness in the suppression of the excessive detrusor activity of the neuropathic bladder hyperreflexia is limited by the widespread side effects and possibly by the contribution to hyperreflexia of the purigenic system. Many other agents said to be more specific for the urinary system such as probanthine, terodilin and oxybutynin have been used, but they also suffer from either low clinical efficacy or cause excessive side effects. Oxybutynin has been administered intravesically with success but is relatively expensive to produce and currently is not yet freely available for clinical use.

This study was designed to test the effectiveness of atropine because it is an antimuscarinic agent whose principle action in the lower urinary tract is at the neuromuscular junction. Furthermore it is widely available in a liquid form suitable for intravesical administration and is inexpensive to produce.

Five patients in this study expelled the atropine soon after it was instilled. Such a high rate of treatment failure will obviously severely restrict the application of intravesical therapy unless it can be reduced. In a study on intravesical therapy with oxybutynin only one of 12 patients expelled the solution. In that study the solution was warmed. ${ }^{4}$ We purposely did not warm the solution as our ultimate intention is for patients to self administer it. As many of the patients are disabled we wished to make the procedure as simple as possible. It is not possible to be certain from these observations that temperature actually plays a vital role in the problem of expulsion of the solution. Other possible factors may also need consideration such as the volume of diluent and speed of instillation. Further investigation of the factors leading to expulsion is necessary. However in patients who do expel the solution it may be sensible to try again with a warmed solution before discarding the technique.

In the seven of the 12 patients who retained the atropine instillation there were increases in cystometric capacity, volume at which leakage first occurred, and the volume at which the first detrusor contraction occurred which were large enough to suggest that patients would have symptomatic improvement in incontinence. Furthermore, this study demonstrated a decrease in the maximum hyperreflexic detrusor contraction which may help to reduce the potential long term complications.

Though we did not show a significant change in end fill pressures or compliance, the changes in compliance came close to being significant and the number of patients was small.

These changes occurred without any discernible side effects. We therefore believe that there is a role for intravesical atropine in the treatment of the neuropathic bladder though many questions and potential problems remain.

Though we have shown a response to a dosage of $6 \mathrm{mg}$ we have not established a dose-response curve. It may be feasible to administer much larger doses as we observed no evidence of any systemic effect. The duration of action has also not been established and therefore the optimal frequency of administration is as yet unknown. If atropine is acting as a competitive antagonist at the neuromuscular junction then its action may rapidly diminish as urine is formed and the local concentration falls.

Though atropine sulphate is supplied in liquid form it is currently distributed in $1 \mathrm{ml}$ vials each containing $0.6 \mathrm{mg}$ atropine. Therefore 10 vials are needed for the dose of $6 \mathrm{mg}$ which was used in this study. Though the cost of each ampoule is only 33p (British National Formulary 1993, No. 22) $)^{12}$ if 10 ampoules are used then a $6 \mathrm{mg}$ dose costs $£ 3.30$, whereas $5 \mathrm{mg}$ oxybutynin chloride tablets cost $£ 28.67$ for a packet of 100 (British National Formulary 1993) giving an individual dose cost of 29p. Most of the cost for atropine is due to packaging and could be substantially reduced if a single large dose ampoule was available. If this is to be self administered by patients who are disabled then perhaps it could be produced in a form more convenient for self administration. Taking this one step further if the temperature of the solution does prove to be important then perhaps a squeezable bag which can be worn next to the skin to attain body temperature prior to use might be a workable arrangement for the disabled patient.

Finally a major obvious drawback for treatment with any intravesical agent is the necessity for catheterisation. This may preclude the more widespread application of intravesical agents to such conditions as primary detrusor instability where patients are less likely to be catheterising.

This study has shown atropine to have a significant effect on detrusor function in humans when administered intravesically, with no side effects. Though this suggests that intravesical atropine may have a role in the management of detrusor hyperreflexia further studies should be performed over longer periods to assess potential clinical benefits.

\section{References}

1 Ukimura O. Effects of intravesically administered anticholinergics, beta-adrenergic stimulant and alpha-adrenergic blocker on bladder function in unanaesthetised rats. Tohoku J Exp Med 
1993; 170: 251-260.

2 Kato $\mathrm{K}$ et al. In vitro intravesical instillation of anticholinergic, antispasmodic and calcium blocking agents to decrease bladder contractility. Urol Int 1991; 47 (Suppl 1): 36-38.

3 Kato $\mathrm{K}$ et al. In vitro intravesical instillation of anticholinergic, antispasmodic and calcium blocking agents (rabbit whole bladder model). J Urol 1989; 141: 1471-1475.

4 Brendler CB, Radebaugh LC, Mohler JL. Topical oxybutynin chloride for relaxation of dysfunctional bladders. J Urol 1989; 141: $1350-1352$.

5 Connor JP et al. Early cystometrograms can predict the response to intravesical instillation of oxybutynin chloride in myelomeningocoele patients. J Urol 1994; 151: 1045-1047.

6 Weese DL, Roskamp DA, Leach GE, Zimmern PE. Intravesical oxybutynin chloride: experience with 42 patients. Urology 1993; 41 : 527-530.
7 Greenfield SP, Fera M. The use of intravesical oxybutynin chloride in children with neurogenic bladder. J Urol 1991; 146 (Pt 2): 532-534.

8 Madersbacher H, Jilg G. Control of detrusor hyperreflexia by the intravesical instillation of oxybutynin hydrochloride. Paraplegia 1991; 29: 84-90.

9 Innes RI, Nickerson M. Atropine scopolamine and related antimuscarinic drugs. In: Goodman SL, Gillman A (eds). Pharmacological Basis of Therapeutics (5th edn). New York: Macmillan, 1975: 514-532.

10 Hinderline PH, Gundert-Remy U, Schmidlin O. Integrated pharmacokinetics and pharmacodynamics of atropine in healthy humans. Pharmacokinetics J Pharm Sci 1985; 74: 703-710.

11 International Continence Society. The standardisation of terminology of lower urinary tract function. Scand $J$ Urol Nephrol 1988; Suppl 114. 